\title{
Evaluation and Treatment of Esophageal Achalasia
}

\author{
Marco G. Patti ${ }^{1}$ Fernando A. M. Herbella ${ }^{2}$
}

Accepted: 16 January 2022 / Published online: 29 January 2022

(C) The Author(s) under exclusive licence to Société Internationale de Chirurgie 2022

Esophageal achalasia is a primary esophageal motility disorders of unknown origin. While in the past a long delay was often present between the onset of symptoms and the diagnosis, today physicians are more aware about achalasia and the disease is diagnosed at an earlier stage. In addition to the classis tests such as the barium swallow and the upper endoscopy, the high-resolution manometry has brought a more precise diagnosis and prognostic value based on its classification in achalasia types I, II, and III.

Today there are excellent options for treatment such as a laparoscopic Heller myotomy with partial fundoplication and POEM. However, it is important to remember that achalasia is never cured and that treatment is palliative. As such, and particularly when treating young patients, it is to be expected that symptoms will recur over a lifetime and that additional intervention is needed. The key for success is the evaluation of patients by a multidisciplinary team composed by radiologists, gastroenterologists, and surgeons with the goal of tailoring treatment to the individual patient and available expertise.

In this symposium, the World Journal of Surgery has brought together a team of experts who have discussed all the key aspects of this disease, from clinical presentation and evaluation to the initial therapeutic approach and treatment of the recurrent symptoms.

The Guest Editors,

Marco G. Patti, MD.

Fernando AM Herbella, MD.

Publisher's Note Springer Nature remains neutral with regard to jurisdictional claims in published maps and institutional affiliations.
Marco G. Patti

Marco.patti@gmail.com

1 Department of Surgery, University of Virginia, Charlottesville, VA, USA

2 Department of Surgery, Universidade Federal de Sao Paulo Escola Paulista de Medicina, Sao Paulo, SP, Brazil 